\title{
Analysis of DNA methylation acquisition at the imprinted DIk1 locus reveals asymmetry at CpG dyads
}

\author{
Alyssa Gagne ${ }^{\dagger}$, Abigail Hochman ${ }^{\dagger}$, Mahvish Qureshi, Celia Tong, Jessica Arbon, Kayla McDaniel \\ and Tamara L Davis
}

\begin{abstract}
Background: Differential distribution of DNA methylation on the parental alleles of imprinted genes distinguishes the alleles from each other and dictates their parent of origin-specific expression patterns. While differential DNA methylation at primary imprinting control regions is inherited via the gametes, additional allele-specific DNA methylation is acquired at secondary sites during embryonic development and plays a role in the maintenance of genomic imprinting. The precise mechanisms by which this somatic DNA methylation is established at secondary sites are not well defined and may vary as methylation acquisition at these sites occurs at different times for genes in different imprinting clusters.
\end{abstract}

Results: In this study, we show that there is also variability in the timing of somatic DNA methylation acquisition at multiple sites within a single imprinting cluster. Paternal allele-specific DNA methylation is initially acquired at similar stages of post-implantation development at the linked D/k1 and Gt/2 differentially methylated regions (DMRs). In contrast, unlike the Gt/2-DMR, the maternal DIk1-DMR acquires DNA methylation in adult tissues.

Conclusions: These data suggest that the acquisition of DNA methylation across the D/k1/Gt/2 imprinting cluster is variable. We further found that the DIk1 differentially methylated region displays low DNA methylation fidelity, as evidenced by the presence of hemimethylation at approximately one-third of the methylated CpG dyads. We hypothesize that the maintenance of DNA methylation may be less efficient at secondary differentially methylated sites than at primary imprinting control regions.

Keywords: Genomic imprinting, DNA methylation, D/k1, Secondary DMR, Epigenetics

\section{Background}

Genomic imprinting in mammals results in the monoallelic expression of approximately 150 genes [1,2]. The majority of these imprinted genes are found in clusters distributed throughout the mammalian genome, with each cluster containing two or more imprinted genes as well as an imprinting control region (ICR) [3]. One common feature of the CpG-rich ICRs is the presence of a gametic, or primary, differentially methylated region (DMR) which generally functions both to identify parental origin and to regulate expression of the imprinted genes within the

\footnotetext{
* Correspondence: tdavis@brynmawr.edu

${ }^{\dagger}$ Equal contributors

Department of Biology, Bryn Mawr College, 101 N. Merion Avenue, Bryn
} Mawr, PA 19010-2899, USA cluster, either directly or indirectly [3]. Establishment of parent of origin-specific DNA methylation at the ICR occurs during gametogenesis and the zygote either inherits a methylated allele from its mother or from its father at fertilization. Differential methylation at the ICR is then maintained throughout development such that the parental alleles can be distinguished from each other and the expression of their adjacent imprinted genes regulated appropriately.

In addition to the differential methylation present at the ICR, some imprinted loci also acquire distinct secondary regions of differential methylation during post-implantation development [4-6]. It has been proposed that the establishment of differential DNA methylation at secondary DMRs could serve as a mechanism for 
maintaining imprinted expression at developmental times when the primary imprinting control region is no longer functioning [6,7]. Support for this hypothesis comes from a recent study of DNA methylation and expression at the imprinted Gpr1-Zdbf2 locus at which the maternally methylated Gpr1 DMR functions as the gametic imprinting mark responsible for establishing paternal allele-specific expression while paternal allele-specific DNA methylation at the secondary $Z d b f 2$ DMR is established after the onset of imprinted $Z d b f 2$ expression [8]. Paternal allele-specific expression of $Z d b f 2$ is maintained after DNA methylation at the Gpr1 DMR becomes biallelic, suggesting that the paternally methylated secondary $Z d b f 2$ DMR functions to maintain monoallelic expression at this locus. Furthermore, biallelic methylation at the Zdbf2 DMR in offspring derived from Dnmt3L $L^{\text {mat-/- }}$ mothers correlated with biallelic expression of $Z d b f 2$. While the exact mechanism responsible for the parental allele-specific acquisition of DNA methylation at secondary DMRs has not yet been determined, it is clear that there is a relationship between the epigenetic states at primary and secondary DMRs $[9,10]$.

The majority of secondary DMRs found at imprinted genes are methylated on the paternally-inherited allele, suggesting that there may be a common mechanism responsible for establishing secondary imprinting marks. At the same time, it is clear that not all secondary DMRs are acquired at the same developmental stage. Paternal allele-specific DNA methylation is established at Gtl2 prior to 6.5 days post coitum (d.p.c.), at $C d k n 1 c$ between 7.5 and 9.5 d.p.c. and at $I g f 2 r$ region 1 during late embryogenesis [7,11-13]. Gtl2, Cdkn1c, and Igf2r are located on mouse chromosomes 12,7 , and 17 , respectively. DNA methylation at secondary DMRs has generally been shown to affect the expression of a single adjacent imprinted gene, rather than the expression of the entire imprinting cluster [6,7]. Therefore, it is possible that the same molecular machinery is used to establish DNA methylation at these sites and that the difference in temporal acquisition reflects the time at which it becomes critical to maintain monoallelic expression for each imprinted gene.

The Dlk1-Dio3 cluster of imprinted genes spans $1 \mathrm{Mb}$ on mouse chromosome 12 and contains three paternally expressed protein-coding genes (Dlk1, Rtl1, and Dio3), multiple maternally expressed untranslated RNAs (including Gtl2), and at least three DMRs that are methylated on the paternal allele [14-18]. The IG-DMR, located between $D l k 1$ and Gtl2, functions as the ICR on the unmethylated maternally inherited allele [19]. Secondary DMRs have been identified at the promoter of Gtl2 and in exon 5 of $D l k 1$ [5]. Evidence suggests that the Gtl2-DMR has a functional role; studies of the mouse Gtl2-DMR and its human homolog, MEG3-DMR, indicate that methylation of this region directly influences expression in cis $[10,20,21]$. Although the functional role of differential methylation at $D l k 1$ has not been determined, both the Gtl2- and Dlk1-DMRs become methylated on the paternal allele following fertilization, and the Gtl2-DMR has been shown to acquire paternal allele-specific methylation during early post-implantation development, between embryonic days 3.5 and 6.5 [5,11]. Since these two DMRs are located within the same imprinting cluster, we hypothesized that the acquisition of paternal allele-specific DNA methylation at these secondary DMRs would be coordinately controlled. We tested this hypothesis by examining the methylation status of the Dlk1-DMR throughout development. We found that the Dlk1-DMR acquires paternal allele-specific methylation during embryogenesis and that the methylation pattern remains dynamic in late embryonic development and into adulthood. Furthermore, our analysis of DNA methylation on the complementary strands of the Dlk1-DMR illustrates the unexpectedly fluid nature of DNA methylation at this locus.

\section{Results}

The Dlk1-DMR acquires paternal allele-specific DNA methylation during post-fertilization development

Previous research illustrated that somatic mouse tissues exhibit paternal allele-specific DNA methylation at the Dlk1-DMR that is acquired after fertilization $[5,14,15]$. To elucidate the temporal acquisition of paternal allelespecific DNA methylation at the $D l k 1$-DMR following fertilization, we assessed the DNA methylation status on both the paternal and maternal $D l k 1$ alleles at various stages of mouse development.

All of our experiments were conducted using $F_{1}$ hybrid tissues collected from crosses between C57BL/6 (B6) and a specially derived strain containing Mus musculus castaneus-derived sequences from chromosome 12 on an otherwise C57BL/6 genetic background (CAST12) [11]. We identified a single nucleotide polymorphism between the B6 and CAST12 strains in a 386 bp CpG island located at the $5^{\prime}$ end of $D l k 1$ exon 5 (http://www.ebi.ac.uk/Tools/ emboss/cpgplot/index.html) [11]. The identified SNP was a C-to-T transition at base pair position 109,459,746 (GenBank: NC_000078.6), preventing us from definitively assigning parental origin following bisulfite mutagenesis and sequencing of the top strand of DNA, since unmethylated cytosines would ultimately be replaced by thymines. Therefore, we modified our approach by covalently attaching the top and bottom strands via a hairpin linker, which allowed us to identify parental origin based on the G-to-A transition on the bottom strand (Figure 1D; see Methods). This approach had the additional advantage of yielding DNA methylation data for complementary CpG dinucleotides, allowing us to determine the level of homo- versus hemimethylation within this region. We used this approach to analyze the methylation status of 16 of the 29 CpGs located within the Dlk1 CpG island (Figure 1C). 


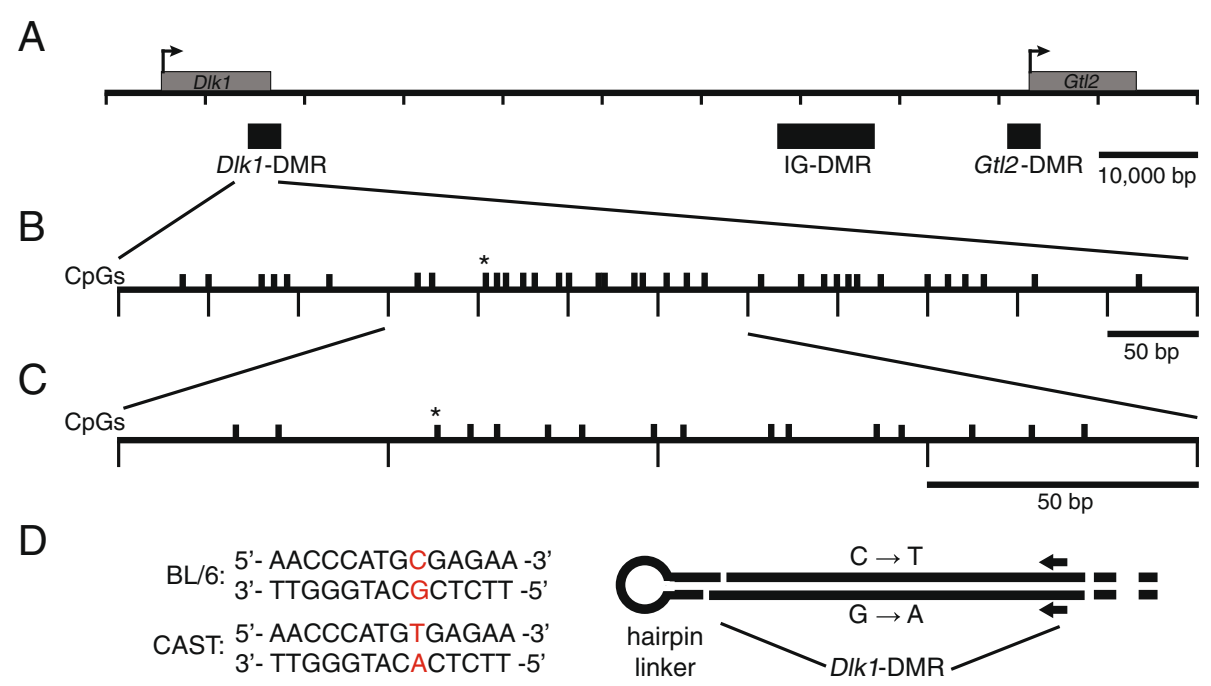

Figure 1 Schematic of Dlk1-Gt/2 imprinting cluster and regions analyzed. (A) D/k1-Gt/2 imprinting cluster, including transcriptional start sites (arrows), transcription units (gray boxes) and differentially methylated regions (black boxes). (B, C) The 600 bp (B) and 220 bp (C) regions of the D/k1-DMR analyzed by bisulfite mutagenesis and DNA sequencing in this study, corresponding to positions 109,459,577-109,460,173 and 109,459,680-109,459,900, NC_000078.6, respectively. The C/T polymorphism (*) between C57BL/6 J and Mus musculus castaneus is located at 109,459,746. (D) Sequence flanking the $\mathrm{C} / T$ polymorphism (red text) and schematic representing ligation of the hairpin linker to Bgll-digested genomic DNA. The hairpin linker was designed to anneal to itself, forming a hairpin structure, and to the 3' overhang generated following Bgll digestion. Ligation of the hairpin linked to Bgll-digested DNA results in the covalent attachment of the complimentary strands of DNA. Primers (block arrows) were designed to anneal to the bisulfite-mutagenized genomic DNA in order to amplify the region of interest.

We confirmed that adult sperm DNA contains very low levels of DNA methylation at the $D l k 1$-DMR (Figure 2A). Therefore, any paternal allele-specific methylation observed in somatic tissues must be acquired during post-fertilization development. To determine when DNA methylation is acquired at the $D l k 1$-DMR, we analyzed the methylation status at the $D l k 1$-DMR during early embryonic development. We were unable to scale down the hairpin linker approach for use with the limited amount of material collected from 3.5 d.p.c. blastocysts and 6.5 d.p.c. embryos. Therefore, for these developmental stages we utilized a traditional bisulfite mutagenesis approach to analyze the DNA methylation status at $36 \mathrm{CpG}$ sites, including all 29 sites contained within the $D l k 1$ CpG island and all 16 sites analyzed using the hairpin linker employed for analysis of DNA derived from older embryonic, neonatal, and adult tissue (Figure 1). We observed an absence of DNA methylation on both the paternal and maternal alleles in 3.5 d.p.c. blastocysts, indicating that the paternal $D l k 1$ allele does not acquire methylation during preimplantation development (Figure 2B). By 6.5 d.p.c., the paternal $D l k 1$ allele has acquired DNA methylation (Figure 2C). We assessed the significance of these results using a Mann-Whitney $U$ test and found that there was a statistically significant difference in the median level of DNA methylation on the paternal alleles of 3.5 vs. 6.5 d.p.c. embryos $(P<0.0001)$. Although the level of
DNA methylation on maternal alleles also increases significantly between 3.5 and 6.5 d.p.c. $(P=0.0023)$, the level of DNA methylation at the paternal Dlk1-DMR in 6.5 d.p.c. embryos is significantly higher than the level of methylation on maternal alleles $(P=0.0025)$, illustrating that differential DNA methylation has been established at the $D l k 1$-DMR by 6.5 d.p.c. All of the raw data used to conduct the Mann-Whitney $U$ tests can be found in Additional file 1.

We next assessed DNA methylation in 7.5 to 9.5 d.p.c. embryos. While the average level of DNA methylation is somewhat variable in 6.5 to 9.5 d.p.c. embryos (Figure 2C-F), neither the variation observed between the paternal alleles at different developmental stages nor between the maternal alleles at different developmental stages was significant, although the paternal and maternal alleles remain different from each other. Average levels of DNA methylation for each of the parental alleles at all developmental stages analyzed are presented in Table 1; this information, along with medians and IQ ranges can be found in Additional file 2 . These results demonstrate that the paternal allelespecific DNA methylation that is established at the Dlk1-DMR prior to 6.5 d.p.c. is maintained during early embryonic development. The timing of DNA methylation acquisition at the $D l k 1-\mathrm{DMR}$ is similar to that which we and others have observed at the paternal Gtl2-DMR [11,12], suggesting that the acquisition 

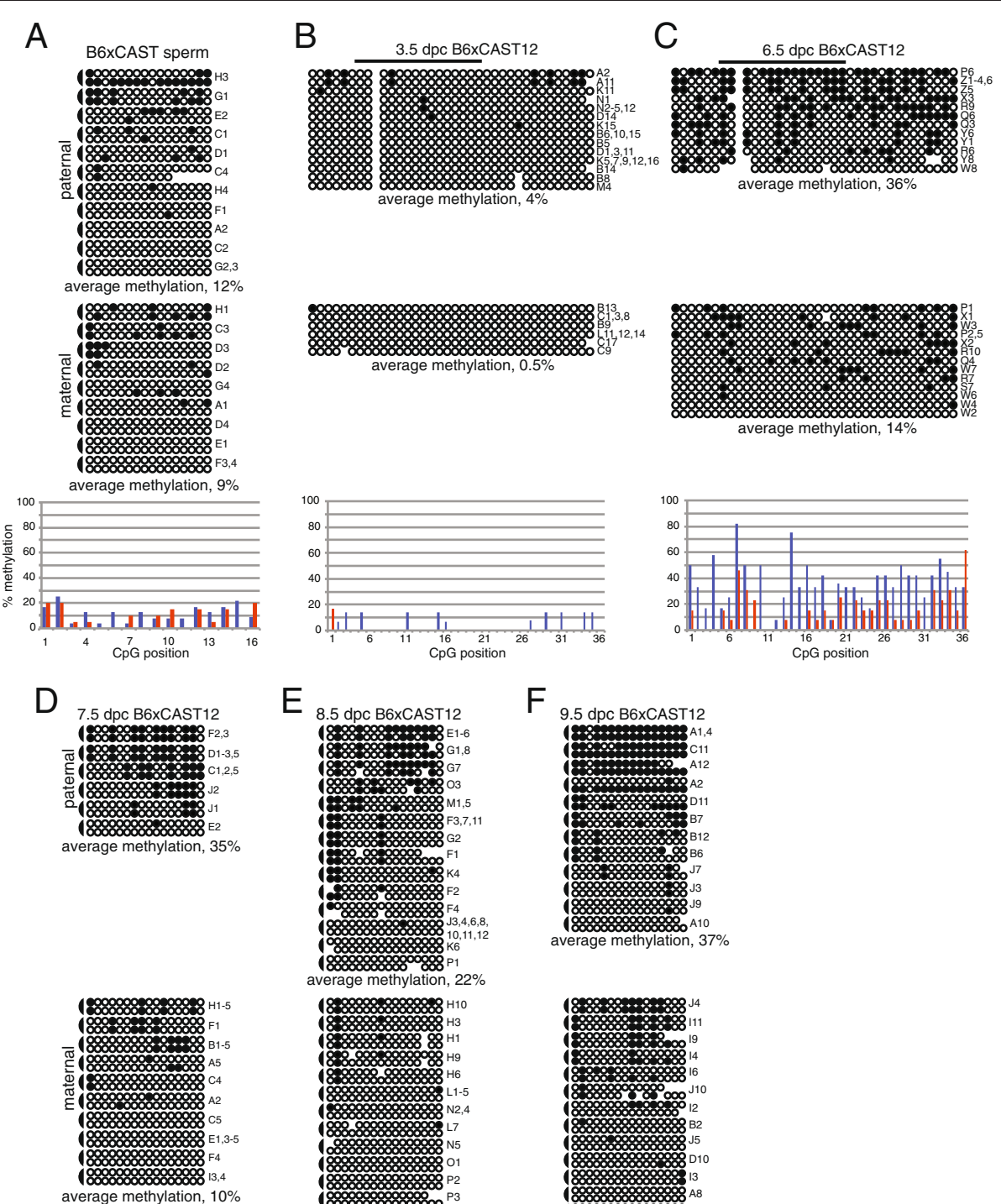

$\mathrm{E}$
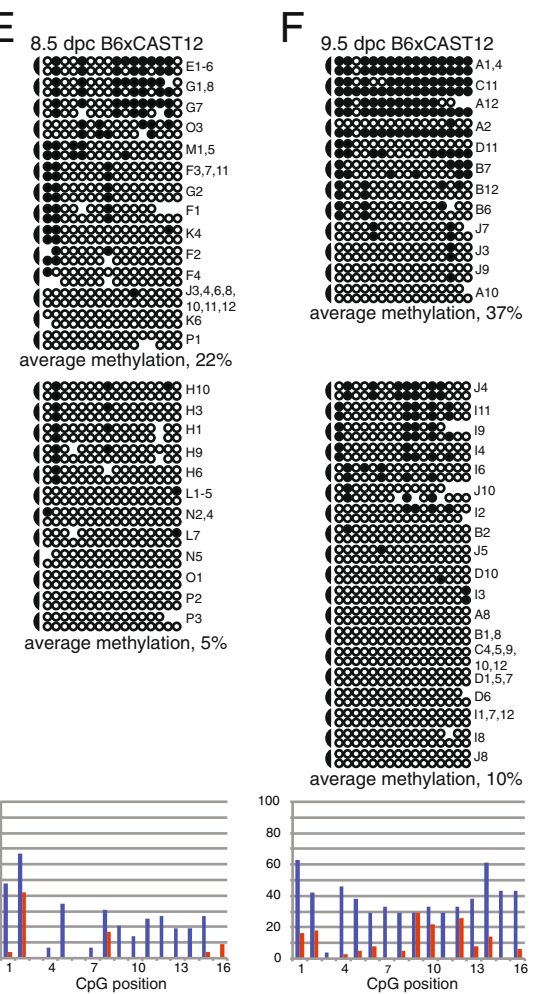

Figure 2 Paternal allele-specific DNA methylation is acquired during post-implantation development. (A) Bisulfite mutagenesis and sequencing of DNA from B6 $\times$ CAST F $F_{1}$ hybrid spermatozoa. (B-F) Bisulfite mutagenesis and sequencing of DNA from B6 $\times$ CAST12 $F_{1}$ hybrid 3.5 to 9.5 d.p.c. embryos. A hairpin linker approach was used to analyze the DNA methylation status of 32 potentially methylated CpG dinucleotides in sperm and 7.5 to 9.5 d.p.c. embryos. Individual circles represent one of the 32 potentially methylated CpG dinucleotides, and each paired row of circles represents the complimentary strands of an individual subclone; semi-circles to the left connect the complimentary strands. A traditional bisulfite mutagenesis approach was utilized to analyze 36 CpG sites located on the bottom, antisense strand in 3.5 d.p.c blastocysts and 6.5 d.p.c. embryos; the analyzed region includes all 29 sites contained within the DIk1 CpG island and all 16 sites analyzed using the hairpin linker (black bar). The gap in the paternal strands represents the polymorphic site, which is not a CpG dinucleotide in Mus musculus castaneus-derived DNA. Filled circles represent methylated cytosines, open circles represent unmethylated cytosines, absent circles represent ambiguous data. Labels to the right identify the PCR subclone analyzed; letters represent independent amplification reactions, while numbers represent individual subclones. The level of methylation ( $y$ axis) on the paternal (blue) and maternal (red) strands was quantified by dividing the number of methylated residues at each CpG site by the total number of sites analyzed at that position. 


\begin{tabular}{|c|c|c|}
\hline Genomic DNA sample & $\begin{array}{l}\% \text { methylation, } \\
\text { paternal alleles }\end{array}$ & $\begin{array}{l}\% \text { methylation, } \\
\text { maternal alleles }\end{array}$ \\
\hline B6xCAST adult sperm & $12 \%$ & $9 \%$ \\
\hline 3.5 d.p.c. B6xCAST12 embryo & $4 \%$ & $0.5 \%$ \\
\hline 6.5 d.p.c. B6xCAST12 embryo & $36 \%$ & $14 \%$ \\
\hline 7.5 d.p.c. B6xCAST12 embryo & $35 \%$ & $10 \%$ \\
\hline 8.5 d.p.c. B6xCAST12 embryo & $22 \%$ & $5 \%$ \\
\hline 9.5 d.p.c. B6xCAST12 embryo & $37 \%$ & $10 \%$ \\
\hline 14.5 d.p.c. B6xCAST12 embryo & $27 \%$ & $5 \%$ \\
\hline 14.5 d.p.c. CAST12xB6 embryo & $21 \%$ & $3 \%$ \\
\hline 17.5 d.p.c. CAST12xB6 liver & $74 \%$ & $18 \%$ \\
\hline 5 d.p.p. B6xCAST12 liver & $46 \%$ & $11 \%$ \\
\hline 5 d.p.p. CAST12xB6 liver & $62 \%$ & $24 \%$ \\
\hline 6 d.p.p. B6xCAST12 lung & $45 \%$ & $8 \%$ \\
\hline 5 d.p.p. CAST12xB6 lung & $44 \%$ & $14 \%$ \\
\hline Adult B6xCAST12 liver & $74 \%$ & $57 \%$ \\
\hline Adult CAST12xB6 liver & $58 \%$ & $41 \%$ \\
\hline Adult B6xCAST12 lung & $18 \%$ & $6 \%$ \\
\hline Adult CAST12xB6 lung & $53 \%$ & $32 \%$ \\
\hline
\end{tabular}

Medians and IQ ranges for each of the average levels of DNA methylation are presented in Additional file 2. In addition, a Kruskal-Wallis test was used to assess overall variation among paternal alleles and among maternal alleles. Significant variation was observed among both the paternal alleles $(P=8.335 \mathrm{e}-13)$ and the maternal alleles $(P=1.844 \mathrm{e}-12)$.

of DNA methylation across the $D l k 1 / G t l 2$ locus may be coordinately controlled.

\section{DNA methylation patterns at the Dlk1-DMR are dynamic during development}

We next examined the DNA methylation status at the Dlk1-DMR in mid- and late-gestation embryos to investigate the progression of DNA methylation acquisition during later developmental stages. The level of methylation we observed on the paternal alleles of 14.5 d.p.c. embryos was similar to the earlier embryos (Figure 3A; Table 1), and statistically significant differences were not observed between 6.5 and 9.5 d.p.c. embryos versus 14.5 d.p.c. embryos. In contrast, $75 \%$ of the CpGs were methylated on paternal alleles derived from 17.5 d.p.c. liver (Figure 3B), and the median level of DNA methylation at this stage was significantly higher when compared to $6.5,7.5,8.5,9.5$, and 14.5 d.p.c. embryos $(P=0.0191$, $0.0435,0.0018,0.0309$, and 0.0005 , respectively). In contrast, no statistically significant differences were detected when DNA methylation levels on the maternal alleles of 17.5 d.p.c. liver were compared to maternal alleles derived from earlier embryos. These data indicate that the DNA methylation status of the paternal Dlk1-DMR continues to be labile into late embryogenesis.

It had previously been reported that the extent of DNA methylation on the maternal and paternal alleles of Dlk1 varied in different tissues [5]. We therefore examined the methylation status at the Dlk1-DMR in stage-matched neonatal liver and lung tissues derived from reciprocal crosses between B6 and CAST12 mice. We chose liver and lung as representative tissues for this analysis as these tissues exhibit low and high levels of Dlk1 expression during perinatal development, respectively [5]. We found that the paternally inherited allele had a significantly higher level of DNA methylation than the maternally inherited allele in both B6xCAST12 and CAST12xB6 tissues $(P=0.001$, liver; $P<0.0001$, lung; Figure $3 C, D)$, consistent with previously obtained data derived from DNA methylation analyses of 18.5 d.p.c. uniparental disomic (UPD) 12 liver and lung tissues [5]. In addition, the median levels of DNA methylation on paternal alleles derived from neonatal liver and lung were significantly higher than the median levels in 14.5 d.p.c. embryos $(P=0.0016$ and 0.004 , respectively), indicating that the DNA methylation level continues to increase on the paternal allele during development. However, we did not detect statistically significant differences in the DNA methylation patterns of neonatal liver versus lung, demonstrating that the methylation status of Dlk1 in these tissues is not different at this developmental stage.

Surprisingly, when we analyzed DNA derived from adult B6xCAST12 liver, we found high levels of methylation on both the paternal (74\%) and maternal alleles (57\%) (Figure 4A). We hypothesized that the maternal Dlk1 allele may acquire methylation at a later stage of development or that the hypermethylation we observed on the maternal allele was an adult tissue-specific pattern. We therefore examined the methylation pattern in DNA derived from adult CAST12xB6 liver as well as adult B6xCAST12 and CAST12xB6 lung. We observed relatively high levels of DNA methylation at the Dlk1-DMR on both parental alleles in adult CAST12xB6 liver, consistent with the DNA methylation profile we observed in adult B6xCAST12 liver (Figure 4A, B), supporting the hypothesis that acquisition of DNA methylation on the maternal $D l k 1$ allele occurs during a later stage of development. While the CAST12xB6 adult lung tissue also displayed high levels of DNA methylation on both parental alleles, both the maternal and the paternal alleles of Dlk1 were hypomethylated in adult B6xCAST12 lung tissue (Figure 4C, D). We did not anticipate the significant difference in DNA methylation status that we observed in the B6xCAST12 versus CAST12xB6 lung tissue, as none of the other data we obtained from stage-matched reciprocal crosses (14.5 d.p.c., 5 to 6 days post partum, and adult liver) displayed this variation. However, it is possible 


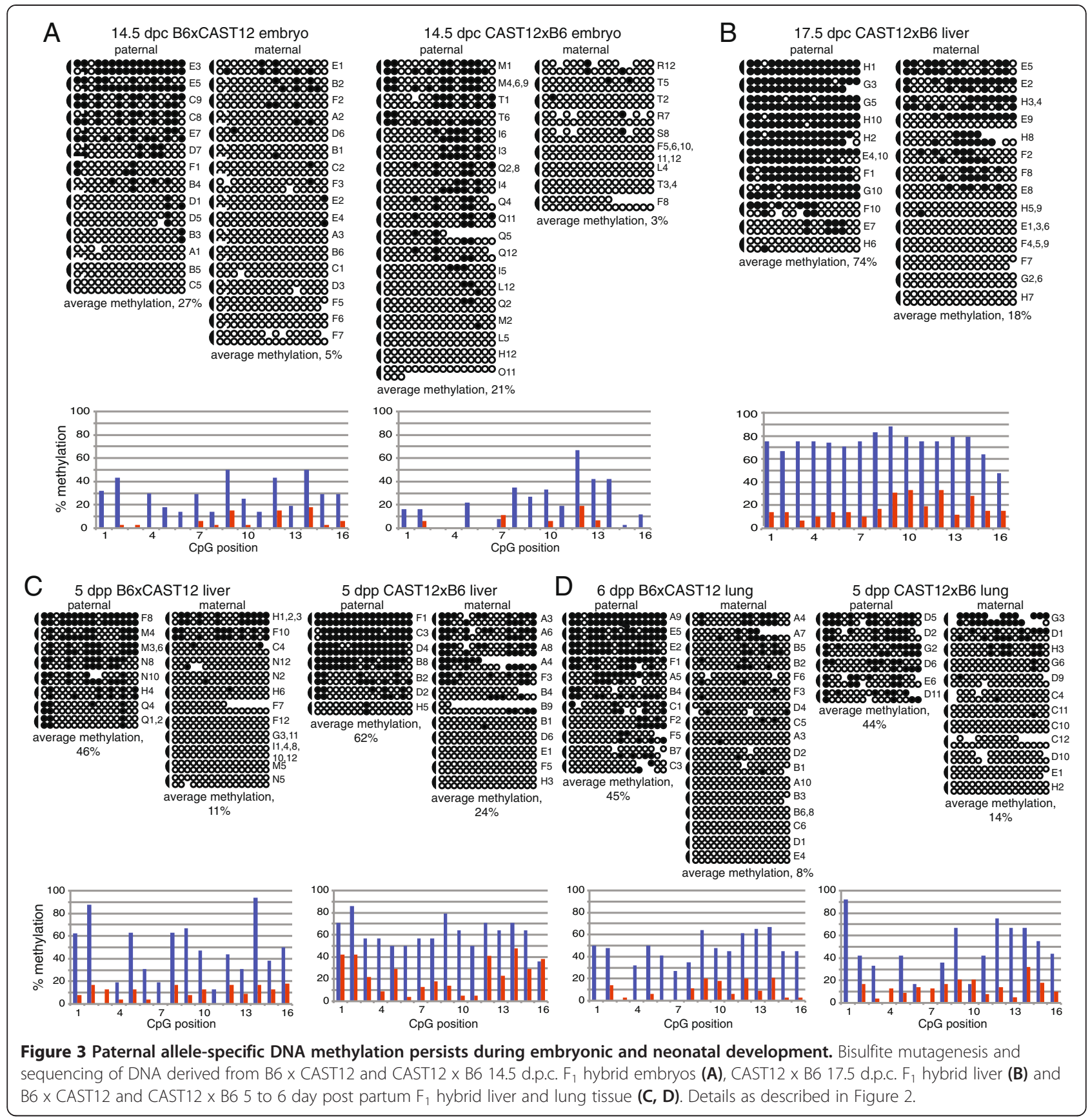

that this experiment uncovered a sensitivity to genetic background at the Dlk1 locus. Further experiments are needed to determine the extent of DNA methylation variation in adult tissues. Regardless, from these results, we conclude that the DNA methylation status of the Dlk1-DMR continues to change during later stages of mouse development.

Our statistical analyses indicate that the low level of DNA methylation observed on the maternal Dlk1-DMR of 6.5 d.p.c. embryos does not change significantly during post-implantation and perinatal development, although it acquired significantly higher levels of DNA methylation in some of the adult tissues analyzed. In contrast, the median level of DNA methylation on the paternal Dlk1-DMR is significantly different in early and mid-gestation embryos when compared either to late embryos or to adult liver, illustrating that the paternal Dlk1-DMR becomes incrementally more methylated over time. Therefore, although the onset of paternal allele-specific DNA methylation acquisition at the Dlk1- and Gtl2-DMRs occurs at a similar time during development, the DNA methylation pattern at the Dlk1-DMR is more labile (Table 1). 


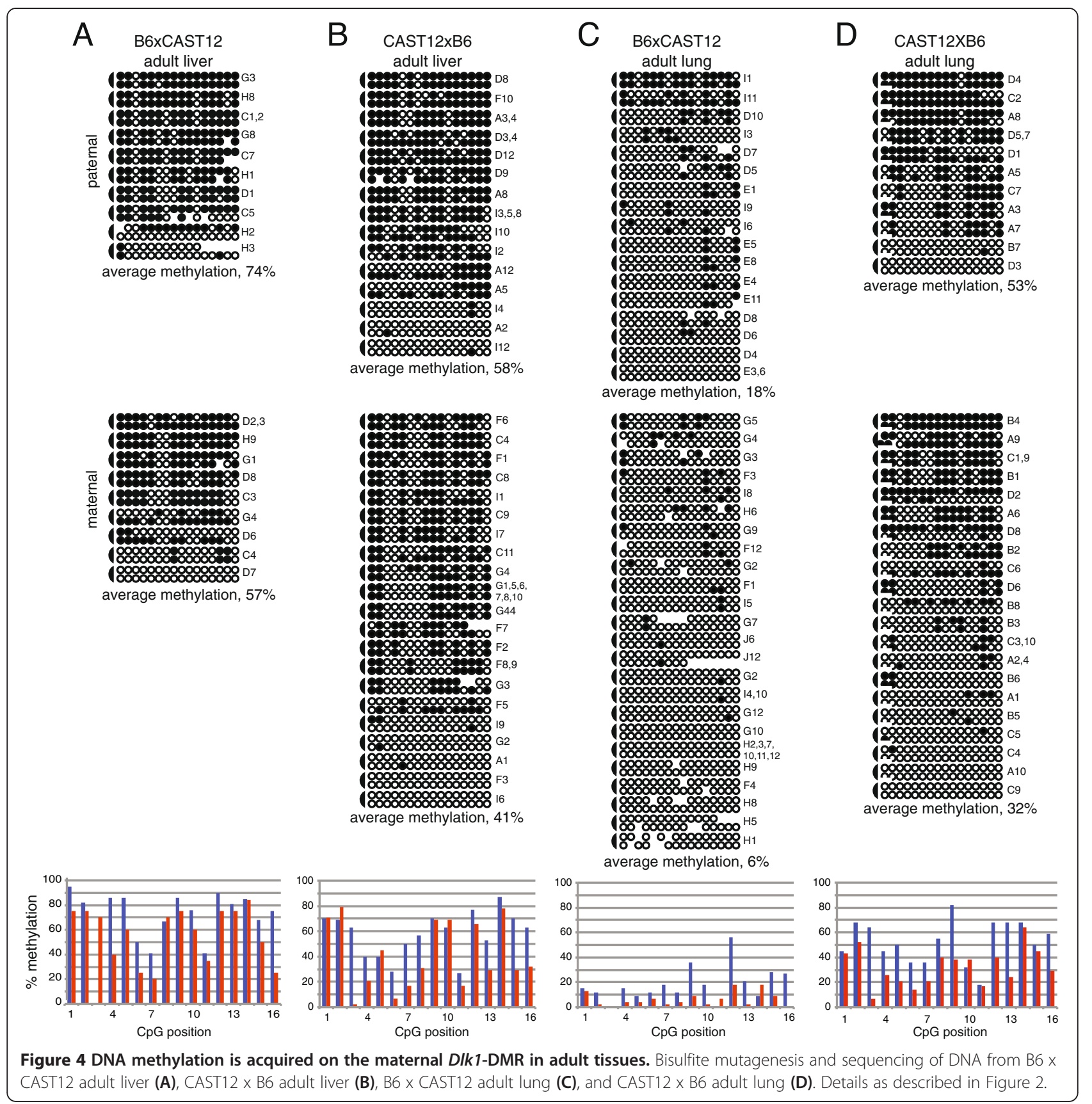

Placental tissue displays biallelic methylation at the Dlk1-DMR

To complete our analysis of the developmental dynamics of DNA methylation at $D l k 1$, we investigated the methylation status in 14.5 d.p.c. B6xCAST12 placenta. Fifty-eight percent of the CpGs were methylated on the paternal alleles and 53.5\% were methylated on the maternal alleles, suggesting that both parental alleles are partially methylated in mouse placenta (Figure 5A). These data are consistent with those previously obtained using a methylation-sensitive southern blot to assess DNA methylation levels on the parental alleles of the $D l k 1-D M R$ in 16.5 d.p.c. placentae [22]. While the median level of DNA methylation on the parental alleles in 14.5 d.p.c. placenta is significantly different from the level observed in the corresponding 14.5 d.p.c. embryo ( $27 \%$ and $5 \%$ for the paternal and maternal alleles, respectively; $P<0.0005)$, previous research has shown that the Gtl2-DMR is methylated on both parental alleles in 6.5, 7.5, and 16.5 d.p.c. extraembryonic tissues, and it has been suggested that both the regulation of the non-coding RNAs and the maintenance of DNA methylation in the Dlk1-Dio3 imprinting cluster 


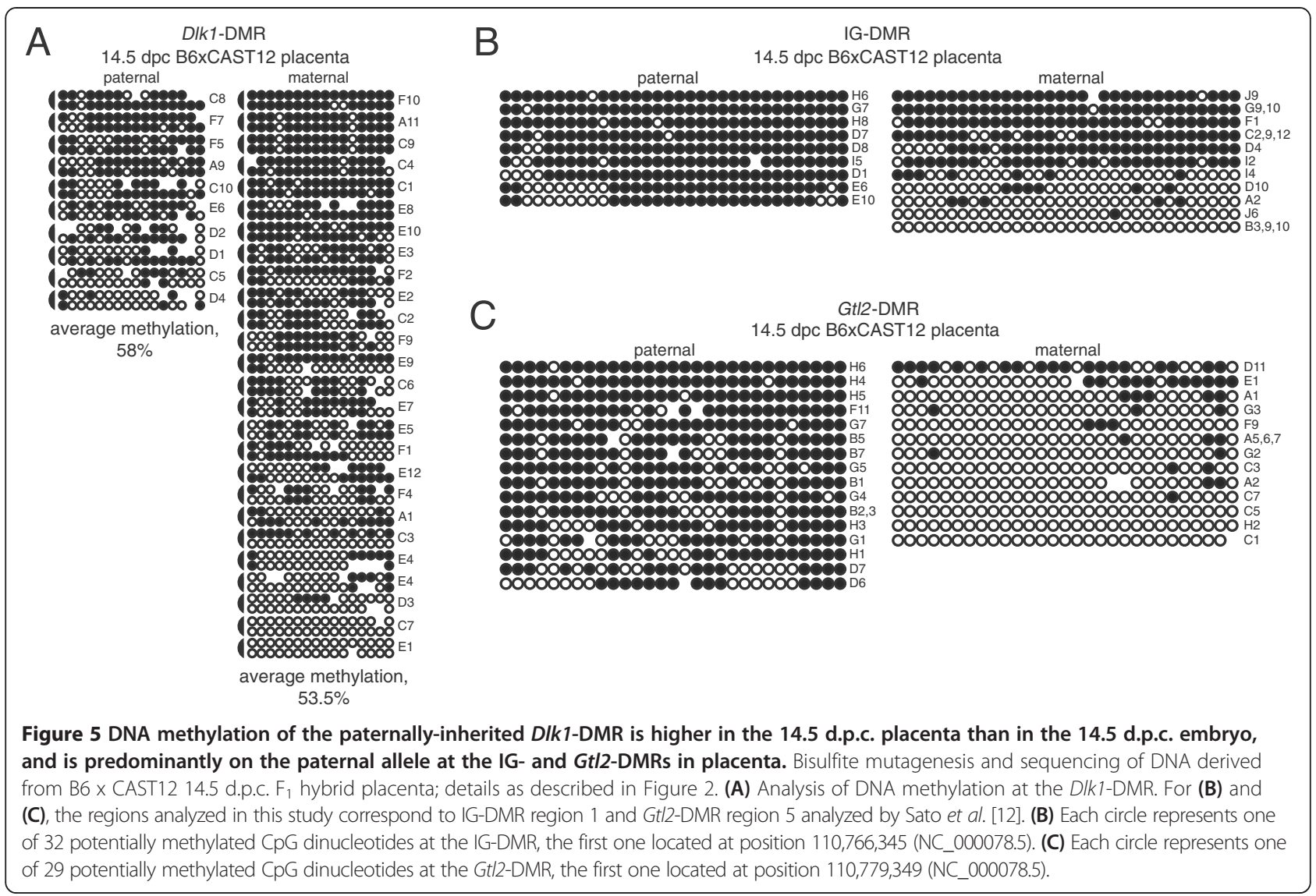

differs in embryonic versus extraembryonic tissue $[12,15,22]$.

Our analysis of methylation at the Dlk1-DMR was complicated by the fact that we did not separate the maternal component of the placenta from the embryonic component. Therefore, while paternal CAST12 alleles must be derived from the embryonic component of the placenta, B6 alleles could derive from the maternal allele in the embryonic component of the placenta or from either of the parental alleles in the maternal component. To assess the relative proportion of embryonic versus maternally-derived B6 DNA in our placental samples, we analyzed the methylation status at the IG-DMR, which has been shown to remain differentially methylated in extraembryonic tissue and placenta [12,22]. As expected, we detected hypermethylation on the nine paternal IG-DMR alleles we analyzed. In contrast, six of the 11 maternal alleles analyzed were hypermethylated, suggesting that these hypermethylated alleles were derived from the maternal component of the placenta and that the level of DNA methylation we observed on the maternal alleles overestimates the true extent of methylation present on the maternal $D l k 1$ alleles in the placenta (Figure 5B). Interestingly, we observed differential methylation on the parental Gtl2-DMR alleles in the same 14.5 d.p.c. placental samples (Figure 5C); these data are in contrast to those obtained by Sato et al. [12]. and Lin et al. [22], who found similar moderate levels of DNA methylation on both the maternal and paternal Gtl2-DMR in 6.5, 7.5, and 16.5 d.p.c. extraembryonic tissue.

\section{CpG dyads within the Dlk1-DMR display a high level of hemimethylation}

The use of a hairpin linker to covalently attach the complementary strands of DNA prior to bisulfite mutagenesis allowed us to examine the DNA methylation status of complementary sites at $\mathrm{CpG}$ dinucleotides. We analyzed a total of 5,965 CpG dyads in embryonic, neonatal, and adult DNA. A total of 1,953 (32.7\%) of the $\mathrm{CpG}$ dyads analyzed contained methylated cytosines. We observed homomethylation at 1,272 sites $(65.1 \%)$ and hemimethylation at 681 sites (34.9\%). While many of the hemimethylated sites were found on sparsely methylated subclones, hemimethylation was also observed at a higher than expected frequency on densely methylated subclones. For example, among the 55 independent subclones we derived from $\mathrm{BxC}$ and $\mathrm{CxB}$ adult liver DNA, 21 subclones were methylated at $65 \%$ to $100 \%$ of the CpG dinucleotides. The total number of CpG dyads with DNA methylation in these densely methylated subclones was $269,87.4 \%$ of 
which were homomethylated (235) and $12.6 \%$ of which were hemimethylated (34) (Table 2).

\section{Discussion}

Proper regulation of imprinted genes is required for normal growth and development in mammals. Loss of imprinting has been shown to result in developmental disorders and disease such as Beckwith-Wiedemann syndrome, which is associated with fetal growth defects, and Prader-Willi and Angelman syndromes, both of which affect neurological development [23]. The regulation of imprinted gene expression is complex and involves various factors, including epigenetic modifications, such as DNA methylation and histone modifications, as well as the activity of long non-coding RNAs and trans-acting factors such as CTCF [3]. The Dlk1-Dio3 imprinting cluster does not contain CTCF binding sites, and while it does include a maternally expressed long non-coding RNA, Gtl2, it is unlikely that Gtl2 expression regulates the paternally expressed $D l k 1$, as there is limited overlap in the expression patterns of these genes $[24,25]$. In contrast, differentially methylated regions have been shown to play an important role in the regulation of imprinted expression within the Dlk1-Dio3 cluster, highlighting the critical role epigenetic modifications play in the regulation of genomic imprinting. For example, deletion of the imprinting control region, IG-DMR, from the maternal chromosome results in its paternalization [19].

In addition to regulating the expression of imprinted genes in the Dlk1-Dio3 cluster, the IG-DMR also influences the acquisition of paternal allele-specific DNA methylation at the secondary Gtl2-DMR. It has been shown that the methylation status of the Gtl2/MEG3DMR is dependent on the methylation status at the IG-DMR, and that inappropriate hypermethylation of the Gtl2/MEG3-DMR is concordant with loss of expression $[10,20,21]$. These data point to a direct role for secondary DMRs in the regulation of imprinted gene expression, although the observation that secondary

Table 2 Extent of homo- vs. hemimethylation at CpG dyads in densely methylated subclones

\begin{tabular}{|c|c|c|c|c|}
\hline & \multicolumn{2}{|c|}{ BxC9C12 adult liver } & \multicolumn{2}{|c|}{ C9C12xB adult liver } \\
\hline & Paternal & Maternal & Paternal & Maternal \\
\hline $\begin{array}{l}\text { Independent subclones } \\
\text { analyzed }(n)\end{array}$ & 10 & 9 & 15 & 21 \\
\hline $\begin{array}{l}\text { Subclones with }>65 \% \\
\text { methylation }(n)\end{array}$ & 8 & 5 & 8 & 0 \\
\hline Methylated dyads $(n)$ & 95 & 65 & 109 & $\mathrm{~N} / \mathrm{A}$ \\
\hline $\begin{array}{l}\text { Homomethylated dyads } \\
(n, \%)\end{array}$ & 89 (93.7\%) & 54 (83.1\%) & 92 (84.4\%) & N/A \\
\hline $\begin{array}{l}\text { Hemimethylated dyads } \\
(n, \%)\end{array}$ & $6(6.3 \%)$ & $11(16.9 \%)$ & $17(15.6 \%)$ & $\mathrm{N} / \mathrm{A}$ \\
\hline
\end{tabular}

DMRs acquire differential methylation after the onset of imprinted expression has led to the hypothesis that secondary DMRs play a role in the maintenance of imprinted expression rather than its establishment [6-8]. To date, this study is the first to examine the temporal acquisition of DNA methylation at multiple secondary DMRs within the same imprinting cluster. Our data illustrate that the timing of post-fertilization DNA methylation acquisition is coordinated across the Dlk1-Dio3 locus, although methylation at the $D l k 1$ locus appears more labile (data herein) [11].

Paternal allele-specific methylation at the Dlk1-DMR is more variable than at many other imprinted loci, in that the total level of methylation on an individual paternally-inherited allele ranges from $0 \%$ to close to $100 \%$ at essentially all developmental stages analyzed. Some of this variation may be attributed to the pattern of DNA methylation acquisition at this locus, which appears to be dynamic throughout development. It is also possible that tissue-specific differences result in the variable DNA methylation patterns we observed in whole embryos. For example, Dlk1 is expressed at high levels in skeletal muscle, a tissue in which imprinting is relaxed, which could correlate with reduced levels of DNA methylation [12,25]. However, even in tissues that display high levels of total DNA methylation on some paternal alleles, such as adult liver, other paternal alleles show little to no methylation and the reason for these differences is not clear. Furthermore, although there are some correlations, there does not appear to be a direct relationship between the DNA methylation profile at the Dlk1-DMR and Dlk1 expression. In most tissues, Dlk1 expression is restricted to the paternal allele, although there is a relaxation of imprinting in 6.5 d.p.c. embryos and in skeletal muscle, in which $20 \%$ and $17 \%$ of the expression is derived from the maternal allele, respectively $[5,12,15,25] . D l k 1$ is expressed at relatively low levels in early embryos, as compared to the high levels of expression detected in various mid- and late-gestation embryonic tissues such as the pituitary gland, skeletal muscle, liver, and lung $[12,25,26]$. Despite these differences in expression, our analyses illustrated that the median levels of DNA methylation on the paternal allele is not significantly different in 6.5 to 14.5 d.p.c. whole embryos (Figures 2, 3; Table 1). Finally, while Dlk1 expression is downregulated in most tissues during late embryogenesis, there was no direct correlation between DNA methylation and Dlk1 expression levels in tissues derived from 18.5 d.p.c. uniparental disomies [5], nor did we detect a direct correlation in this study. Together, these data suggest that the DNA methylation status at the Dlk1-DMR, located in exon 5, may not play an important role in the regulation of expression at this locus. In contrast, the methylation status of the Gtl2/MEG3-DMR has been shown to directly 
influence expression of Gtl2 in cis, consistent with its location at the Gtl2 promoter [5,10,20,21]. The critical regulatory role of the Gtl2-DMR may explain the maintenance of high average DNA methylation levels at this locus once it has been established $[5,11,12]$. It is possible that DNA methylation at the Dlk1-DMR may reflect a broader, locus-wide epigenetic profile that encompasses both Gtl2 and Dlk1.

\section{The DIk1-DMR displays low methylation fidelity}

The approach we utilized allowed us to analyze the methylation pattern for complementary $\mathrm{CpG}$ dinucleotides within the Dlk1-DMR. To the best of our knowledge, this is the first study to comprehensively examine the methylation status of complementary $\mathrm{CpG}$ dinucleotides at an imprinted gene during development. Of the 1,953 methylated CpG dyads, 1,272 (65.1\%) were homomethylated, while 681 (34.9\%) were hemimethylated. This result was unexpected, as the fidelity with which the maintenance DNA methyltransferase in mouse, Dnmt1, has been shown to be greater than $95 \%[27,28]$. There are several possible reasons to explain some of the hemimethylation we detected. It is likely that some of the hemimethylated sites we observed are a result of hybrid subclones, which have been shown to result as an artifact of PCR amplification following bisulfite mutagenesis [29]. It is also possible that some of the observed hemimethylation is a result of Taq-induced PCR error during amplification. However, these artifacts are unlikely to account for the high level of hemimethylation we detected. Rather, the high level of hemimethylation we observed challenges the idea that Dnmt1 functions with high fidelity at all genomic locations.

A large-scale study analyzing the in vivo regulation of CpG methylation by DNA methyltransferases was recently conducted by Arand et al. [30]. In this study, the authors found relatively high levels of hemimethylated CpGs in embryonic liver, ranging from $16.2 \%$ to $30.6 \%$ of the methylated CpG dyads. Interestingly, this work illustrated the relative stability of homomethylation at the imprinted Snprn and H19 genes, but demonstrated high levels of hemimethylation at the imprinted Igf2 gene (22\%). Analyses of DNA methylation profiles in Dnmt-mutant embryonic stem cells indicated that the DNA methylation profiles at Snprn and H19 were dependent on the activity of Dnmt1 alone, while maintenance of DNA methylation at Igf2 required the coordinated activity of Dnmt1, Dnmt3a, and Dnmt3b, a possible consequence of 5-hydroxymethylcytosine enrichment at the Igf2 DMR [30]. It is therefore possible that the high level of hemimethylation we observed at the Dlk1-DMR may be due to the presence of 5-hydroxymethylcytosine at this locus, preventing high levels of fidelity via Dnmt1. An analysis of methylcytosine versus 5-hydroxymethylcytosine levels at the Dlk1-DMR will address this possibility.
An alternative hypothesis to explain the high level of hemimethylation we observed at the Dlk1-DMR is that there may be a lower level of fidelity associated with the maintenance of DNA methylation at secondary DMRs. Consistent with this hypothesis, a study by $\mathrm{Vu}$ et al. [31] examined DNA methylation on the top and bottom strands of the human Igf2/H19 imprinted region. Vu and colleagues analyzed DNA methylation on the top and bottom strands separately and found uniform levels of methylation present at the primary DMR. In contrast, they observed less uniformity in the methylation of the top and bottom strands at the H19 promoter, which is categorized as a secondary DMR as it loses and then regains paternal allele-specific methylation during preand post-implantation development, respectively [32,33]. Additionally, a more recent survey of differentially methylated regions associated with imprinted genes in humans support this hypothesis. Woodfine et al. [34] reported a higher level of stability for DNA methylation at gametic DMRs than at secondary DMRs. Further examination of CpG dyad methylation patterns at imprinted loci may provide additional insight into the mechanisms responsible for the acquisition and maintenance of DNA methylation at these sites.

\section{Conclusions}

Our analysis of DNA methylation at the mouse Dlk1-DMR illustrates that the acquisition of paternal allele-specific DNA methylation initiates between 3.5 and 6.5 d.p.c., suggesting that epigenetic modifications across the Dlk1-Dio3 imprinting cluster may be coordinately regulated during post-implantation development. The range of DNA methylation levels on individual alleles at the same developmental stage as well as the additional acquisition of DNA methylation on the maternal $D l k 1$ allele in adult tissues suggest that the DNA methylation profile of this secondary DMR is more variable than is commonly seen at imprinted loci. We further observed a high level of hemimethylation at the Dlk1-DMR: 35\% of CpG dyads containing methylated residues were methylated on only one of the two complementary strands. This result is significant because it challenges the idea that Dnmt1 functions with high fidelity at all genomic locations. We hypothesize that the low DNA methylation fidelity we observed is related to the variable DNA methylation profiles at the Dlk1-DMR, and may be a consequence of high levels of 5-hydroxymethylcytosine at this locus. These data provide insight into a novel epigenetic profile that may distinguish primary DMRs from secondary DMRs.

\section{Methods \\ Mice}

C57BL/6 J (B6) and Mus musculus castaneus (CAST) mice were purchased from the Jackson Laboratory. To 
facilitate the isolation of $F_{1}$ hybrid mice, a strain of mice that served as the source of the M. m. castaneus allele (CAST12) was constructed as previously described [11]. Natural matings between B6 and CAST were used to generate $F_{1}$ hybrid males for spermatozoa collection; all other $F_{1}$ hybrid tissues used for bisulfite analyses were generated from natural matings between B6 and CAST12 mice. For all $F_{1}$ hybrid tissues, the maternal allele is located on the left. Ethical approval for procedures involving animals was granted by the Bryn Mawr College Institutional Animal Care and Use Committee, PHS Welfare Assurance Number A3920-01.

\section{DNA purification and bisulfite analysis}

For bisulfite analysis of 3.5 and 6.5 d.p.c. DNA, two to four embryos were pooled prior to digestion with proteinase $\mathrm{K}$. The resulting DNA was subjected to bisulfite mutagenesis using an EZ DNA methylation-direct kit (Zymo Research, cat\# D5020). For all other tissues, genomic DNA extractions were performed either from a pool (four 7.5 d.p.c. embryos) or from single embryos, fetuses, or tissues according to the DNeasy protocol (Qiagen) or using a series of phenol/chloroform extractions as described previously [33], and the complementary strands were covalently attached prior to bisulfite mutagenesis as follows: $0.5 \mu \mathrm{g}$ of genomic DNA was digested with $1 \mu \mathrm{L}$ BglI (NEB, cat\# R0143S) and ligated to $1 \mu \mathrm{g}$ of a phosphorylated hairpin linker (5'-AGCGATGCGTTCGAG CATCGCTCCC-3') [35]. A total of $0.5 \mu \mathrm{g}$ of hairpin linked-ligated DNA was denatured by incubating in freshly prepared $3 \mathrm{M} \mathrm{NaOH}$ for $20 \mathrm{~min}$ at $42^{\circ} \mathrm{C}$, then subjected to bisulfite mutagenesis using an EZ DNA methylation-direct kit, as above. All mutagenized DNAs were subjected to multiple independent PCR amplifications to ensure analysis of different strands of DNA; subclones derived from independent PCR amplifications are distinguished by different letters of the alphabet. Data from multiple individuals at the same developmental stage were combined, as we did not detect variation between biological replicates. The following primer pairs were used for nested amplification of the mutagenized DNA, and were designed to incorporate both the SNP and at least $50 \%$ of the CpG dinucleotides within the $\mathrm{CpG}$ island. All base pair numbers are from GenBank Accession Number NC_000078.6. For the first round of amplification of mutagenized 3.5 and 6.5 d.p.c. DNA, two cycles of $94^{\circ} \mathrm{C}$ for $2 \mathrm{~min}, 52^{\circ} \mathrm{C}$ for $1 \mathrm{~min}, 72^{\circ} \mathrm{C}$ for $1 \mathrm{~min}$ followed by 30 cycles of $94^{\circ} \mathrm{C}$ for $30 \mathrm{~s}, 52^{\circ} \mathrm{C}$ for $1 \mathrm{~min}, 72^{\circ} \mathrm{C}$ for 1 min using primers RDlke5BF3 (5'-CCCCATCTAAC TAATAACTTACA-3')/RDlke5BR3 (5'-GTGTTTAGTAT TATTAGGTTGGTG-3'). For the second round of amplification, 35 cycles of $94^{\circ} \mathrm{C}$ for $30 \mathrm{~s}, 52^{\circ} \mathrm{C}$ for $1 \mathrm{~min}, 72^{\circ} \mathrm{C}$ for $1 \mathrm{~min}$ using primers RDlke5BF4 (5' -
ATTTCTACTACTCTATCCTAACCC-3')/RDlke5BR4 (5' TTAGGATGGTGAAGTAGATGGT-3') yielded a 597 bp product. To amplify mutagenized DNA treated with the hairpin linker, the same reaction conditions were used with the following primers to yield a 464 bp product: first round, RDlke5BR4 (5'-TTAGGATGGTGAAGTA GATGGT-3')/Dlk1e5BR1 (5'-AACTCTTTCATAAACAC CTTCAA-3'); second round, HPDlk1e5F (5'-GTTTAT TTGGGTGTGTTGGAGG-3')/HPDlk1e5R (5'-AAACT CACCTAAATATACTAAAAAC-3'). The following primer pairs were used for nested or semi-nested amplification of IG- and Gtl2-DMRs, as previously described [11]. All base pair numbers are from NC_000078.5. Gtl2 IG-DMR, with the first nucleotide of IG-BS-F1 corresponding to position $110,766,235$ : 30 cycles of $94^{\circ} \mathrm{C}$ for $30 \mathrm{~s}, 52^{\circ} \mathrm{C}$ for $1 \mathrm{~min}, 72^{\circ} \mathrm{C}$ for $1 \mathrm{~min}$, using primers IG-BS-F1/IG-BS-R, followed by 35 cycles using IG-BS-F2/ IG-BS-R and the same cycling conditions as above. Identical reaction conditions were used to amplify the Gtl2-DMR, with the first nucleotide of Gtl2BI4F1 corresponding to position 110,779,293: Gtl2BI4F1/Gtl2BI4R1 followed by Gtl2BI4F2/Gtl2BI4R2. Primer sequences follow. IG-BS-F1, 5'-GTATGTGTATAGAGATATGTTT ATATGGTA-3'; IG-BS-F2, 5'-GTGTTAAGGTATAT TATGTTAGTGTTAGGA-3'; IG-BS-R, 5'-GCTCCAT TAACAAAATAATACAACCCTTCC-3'; Gtl2BI4F1, 5'GAAGAATTTTTTATTTGGTGAGTGG-3'; Gtl2BI4F2, 5'-GTTTGAAAGGATGTGTAAAAATG-3'; Gt12BI4R1, 5'-CAACACTCAAATCACCCCCC-3'; Gtl2BI4R2, 5' -GC CCCCCACATCTATTCTACC-3'. Subcloning of amplified products was achieved using a pGEM-T Easy vector (Promega Corporation, Madison, WI, USA). Sequencing reactions were performed using a Thermo Sequenase Cycle Sequencing Kit (USB Corporation, Cleveland, $\mathrm{OH}, \mathrm{USA}$ ), and reactions were analyzed on a 4300 DNA Analyzer (LI-COR Biosciences, Lincoln, NE, USA). Percent methylation was calculated based on data obtained from both complementary strands.

\section{Identification of $\mathrm{CpG}$ island}

The extent of the CpG island identified by Paulsen et al. [24] was determined using the EMBOSS CpGPlot analyzer (http://www.ebi.ac.uk/Tools/emboss/cpgplot/ index.html), with the following parameters: program = cpgplot, window $=200$, step $=1$, obs $/ \exp =0.6, \operatorname{MinPC}=50$, length $=200$. The position of the CpG island corresponds to nucleotides 109,459,650-109,460,035 (GenBank: NC_000078.6).

\section{Additional files}

Additional file 1: Data used for statistical analyses of DNA methylation levels at the Dlk1-DMR during different stages of mouse development. This file contains the numerical data used to 
perform Kruskal-Wallis and Mann-Whitney $U$ tests. Data from each of the developmental stages are presented in chronological order, as they are in the Results, Figures, and Table 1. Each dataset presents the information for a specific tissue, cross (maternal allele $x$ paternal allele), and parental allele analyzed, as indicated in columns B-D.\% methylation (column E) was calculated by dividing the number of methylated $\mathrm{CpG}$ sites observed in a given subclone (column $\mathrm{A}$ ) by the total number of $\mathrm{CpG}$ sites analyzed within the subclone; the raw data used to make these calculations are found in Figures 2, 3, 4, and 5.

Additional file 2: Average levels of DNA methylation on the paternal and maternal Dlk1-DMR alleles during development, including median values and IQ ranges. This file expands on the information presented in Table 1. In addition to presenting the average levels of DNA methylation at each developmental stage, Additional file 2 contains median values and IQ ranges. Data from each developmenta stage are presented in chronological order, as they are in the Results and Figures.

\section{Abbreviations}

B6: C57BL/6; C or CAST: Mus musculus castaneus; C12 or CAST12: Mus musculus castaneus chromosome 12 on a C57BL/6 background; DMR: Differentially methylated region; d.p.c.: Days post coitum; d.p.p.: Days post partum; ICR: Imprinting control region; PCR: Polymerase chain reaction; UPD: Uniparental disomy.

\section{Competing interests}

The authors declare that they have no competing interests.

\section{Authors' contributions}

MQ participated in experimental design and carried out molecular genetic studies. AG, AH, CT, JA, and KM carried out molecular genetic studies. TLD conceived of the study and experimental design, carried out molecular genetic studies, and wrote the manuscript. All authors read and approved the final manuscript.

\section{Acknowledgements}

We thank Jeanette Bates for her contributions towards this work, Joshua Shapiro for assistance with the statistical analyses, and Michelle Wien and Joshua Shapiro for thoughtful discussion. This work was supported by awards from the Bryn Mawr College Faculty Research Fund and National Science Foundation grant 1157819 to TLD. In addition, AG, AH, MQ, CT, JA, and KM were supported in part by the Bryn Mawr College Summer Science Research program.

Received: 11 February 2014 Accepted: 20 May 2014

Published: 29 May 2014

\section{References}

1. Morison IM, Ramsay JP, Spencer HG: A census of mammalian imprinting. Trends Genet 2005, 21:457-465.

2. Williamson CM, Blake A, Thomas S, Beechey CV, Hancock J, Cattanach BM, Peters J: World Wide Web Site, Mouse Imprinting Data and References. Oxfordshire: MRC Harwell; 2011. http://www.har.mrc.ac.uk/research/ genomic_imprinting/.

3. Bartolomei MS, Ferguson-Smith AC: Mammalian genomic imprinting. Cold Spring Harb Perspect Biol 2011, 3:a002592.

4. Hanel M, Wevrick R: Establishment and maintenance of DNA methylation patterns in mouse Ndn: implications for maintenance of imprinting in target genes of the imprinting center. Mol Cell Biol 2001, 21:2384-2392.

5. Takada S, Paulsen M, Tevendale M, Tsai C-E, Kelsey G, Cattanach BM, Ferguson-Smith AC: Epigenetic analysis of the D/k1-Gt/2 imprinted domain on mouse chromosome 12: implications for imprinting control from comparison with Igf2-H19. Hum Mol Genet 2002, 11:77-86.

6. Bhogal B, Arnaudo A, Dymkowski A, Best A, Davis TL: Methylation at mouse Cdkn1c is acquired during postimplantation development and functions to maintain imprinted expression. Genomics 2004, 84:961-970.

7. John RM, Lefebvre L: Developmental regulation of somatic imprints. Differentiation 2011, 81:270-280.

8. Kobayashi H, Sakurai T, Sato S, Nakabayashi K, Hata K, Kono T: Imprinted DNA methylation reprogramming during early mouse embryogenesis at the Gpr1-Zdbf2 locus is linked to long cis-intergenic transcription. FEBS Lett 2012, 586:827-833.

9. Lopes S, Lewis A, Hajkova P, Dean W, Oswald J, Forné T, Murrell A, Constância M, Bartolomei M, Walter J, Reik W: Epigenetic modifications in an imprinting cluster are controlled by a hierarchy of DMRs suggesting long-range chromatin interactions. Hum Mol Genet 2003, 12:295-305.

10. Kagami M, O'Sullivan MJ, Green AJ, Watabe Y, Arisaka O, Masawa N, Matsuoka K, Fukami M, Matsubara K, Kato F, Ferguson-Smith AC, Ogata T: The IG-DMR and the MEG3-DMR at human chromosome 14q32.2: hierarchical interaction and distinct functional properties as imprinting control centers. PLoS Genet 2010, 6:1-13.

11. Nowak K, Stein G, Powell E, He LM, Naik S, Morris J, Marlow S, Davis TL: Establishment of paternal allele-specific DNA methylation at the imprinted mouse Gt/2 locus. Epigenetics 2011, 6:1012-1020.

12. Sato S, Yoshida W, Soejima H, Nakabayashi K, Hata K: Methylation dynamics of IG-DMR and Gt/2-DMR during murine embryonic and placental development. Genomics 2011, 98:120-127.

13. Stöger R, Kubicka P, Liu C-G, Kafri T, Razin A, Cedar H, Barlow DP: Maternal-specific methylation of the imprinted mouse lgf2r locus identifies the expressed locus as carrying the imprinting signal. Cell 1993, 73:61-71.

14. Takada S, Tevendale M, Baker J, Georgiades P, Campbell E, Freeman T, Johnson MH, Paulsen M, Ferguson-Smith AC: Delta-like and Gt/2 are reciprocally expressed, differentially methylated linked imprinted genes on mouse chromosome 12. Curr Biol 2000, 10:1135-1138.

15. Schmidt JV, Matteson PG, Jones BK, Guan X-J, Tilghman SM: The Dlk1 and Gt/2 genes are linked and reciprocally imprinted. Genes Dev 2000, 14:1997-2002.

16. Yevtodiyenko A, Carr MS, Patel N, Schmidt JV: Analysis of candidate imprinted genes linked to D/k1-Gt/2 using a congenic mouse line. Mamm Genome 2002, 13:633-638.

17. Seitz H, Royo H, Bortolin ML, Lin SP, Ferguson-Smith AC, Cavaille J: A large imprinted microRNA cluster at the mouse Dlk1-Gt/2 domain. Genome Res 2004, 14:1741-1748.

18. Hagan JP, O'Neill BL, Stewart CL, Kozlov SV, Croce CM: At least ten genes define the imprinted Dlk1-Dio3 cluster on mouse chromosome 12qF1. PLoS One 2009, 4:e4352.

19. Lin S-P, Youngson N, Takada S, Seitz H, Reik W, Paulsen M, Cavaille J, Ferguson-Smith AC: Asymmetric regulation of imprinting on the maternal and paternal chromosomes at the Dlk1-Gt/2 imprinted cluster on mouse chromosome 12. Nat Genet 2003, 35:97-102.

20. Sekita Y, Wagatsuma H, Irie M, Kobayashi S, Kohda T, Matsuda J, Yokoyama M, Ogura A, Shuster-Gossler K, Gossler A, Ishino F, Kaneko-Ishino T: Aberrant regulation of imprinted gene expression in Gt12 ${ }^{\text {lacz }}$ mice. Cytogenet Genome Res 2006, 113:223-229.

21. Steshina EY, Carr MS, Glick EA, Yevtodiyenko A, Appelbe OK, Schmidt JV: Loss of imprinting at the Dlk1-Gt/2 locus caused by insertional mutagenesis in the Gt/2 5' region. BMC Genet 2006, 7:44.

22. Lin S-P, Coan P, da Rocha ST, Seitz H, Cavaille J, Teng P-W, Takada S, Ferguson-Smith AC: Differential regulation of imprinting in the murine embryo and placenta by the Dlk1-Dio3 imprinting control region. Development 2007, 134:417-426.

23. Robertson KD: DNA methylation and human disease. Nat Rev Genet 2005, 6:597-610.

24. Paulsen M, Takada S, Youngson NA, Benchaib M, Charlier C, Segers K, Georges M, Ferguson-Smith AC: Comparative sequence analysis of the imprinted $D \mid k 1-G t / 2$ locus in three mammalian species reveals highly conserved genomic elements and refines comparison with the Igf2-H19 region. Genome Res 2001, 11:2085-2094.

25. da Rocha ST, Tevendale M, Knowles E, Takada S, Watkins M, Ferguson-Smith AC: Restricted co-expression of $D / k 1$ and the reciprocally imprinted non-coding RNA, Gt12: implications for cis-acting control. Dev Biol 2007, 306:810-823.

26. Yevtodiyenko A, Schmidt JV: Dlk1 expression marks developing endothelium and sites of branching morphogenesis in the mouse embryo and placenta. Dev Dynam 2006, 235:1115-1123.

27. Hirasawa R, Chiba H, Kaneda M, Tajima S, Li E, Jaenisch R, Sasaki H: Maternal and zygotic Dnmt1 are necessary and sufficient for the maintenance of DNA methylation imprints during preimplantation development. Genes Dev 2008, 22:1607-1616.

28. Vilkaitis G, Suetake I, Klimasauskas S, Tajima S: Processive methylation of hemimethylated $\mathrm{CpG}$ sites by mouse Dnmt1 DNA methyltransferase. J Biol Chem 2005, 280:64-72. 
29. Warnecke PM, Stirzaker C, Song J, Grunau C, Melki JR, Clark SJ: Identification and resolution of artifacts in bisulfite sequencing. Methods 2002, 27:101-107.

30. Arand J, Spieler D, Karius T, Branco MR, Meilinger D, Meissner A, Jenuwein T, $\mathrm{Xu} \mathrm{G}$, Leonhardt $\mathrm{H}$, Wolf $\mathrm{V}$, Walter J: In vivo control of $\mathrm{CpG}$ and non-CpG DNA methylation by DNA methyltransferases. PLoS Genet 2012, 8:e1002750.

31. Vu TH, Li T, Nguyen D, Nguyen BT, Yao X-M, Hu J-F, Hoffman AR: Symmetric and asymmetric DNA methylation in the human IGF2-H19 imprinted region. Genomics 2000, 64:132-143.

32. Tremblay KD, Duran KL, Bartolomei MS: A 5' 2-kilobase-pair region of the imprinted mouse $\mathrm{H} 19$ gene exhibits exclusive paternal methylation throughout development. Mol Cell Biol 1997, 17:4322-4329.

33. Davis TL, Trasler JM, Moss SB, Yang GJ, Bartolomei MS: Acquisition of the H19 methylation imprint occurs differentially on the parental alleles during spermatogenesis. Genomics 1999, 58:18-28.

34. Woodfine K, Huddleston JE, Murrell A: Quantitative analysis of DNA methylation at all human imprinted regions reveals preservation of epigenetic stability in adult somatic tissue. Epigenetics Chromatin 2011, 4:1.

35. Laird CD, Pleasant ND, Clark AD, Sneeden JL, Hassan KMA, Manley NC, Vary JC, Morgan T, Hansen RS, Stöger R: Hairpin-bisulfite PCR: assessing epigenetic methylation patterns on complementary strands of individual DNA molecules. Proc Natl Acad Sci U S A 2004, 101:204-209.

doi:10.1186/1756-8935-7-9

Cite this article as: Gagne et al:: Analysis of DNA methylation acquisition at the imprinted Dlk1 locus reveals asymmetry at $\mathrm{CpG}$ dyads. Epigenetics \& Chromatin 2014 7:9.

\section{Submit your next manuscript to BioMed Central and take full advantage of:}

- Convenient online submission

- Thorough peer review

- No space constraints or color figure charges

- Immediate publication on acceptance

- Inclusion in PubMed, CAS, Scopus and Google Scholar

- Research which is freely available for redistribution 